\title{
Epistaxis caused by nasal septum angioleiomyoma*
}

\section{Gabriele Noreikaite', Sihun Alex Kim², Adelaide Horcher ${ }^{3}$}

' Midwestern University, Chicago College of Osteopathic Medicine, Downers Grove, IL, USA

'Ear Nose Throat and Sinus Surgery, Northwestern Medicine McHenry Hospital, McHenry, IL, USA

3 Pathology, Northwestern Medicine McHenry Hospital, McHenry, IL, USA
Rhinology Online, Vol 3: 221 - 224, 2020

http://doi.org/10.4193/RHINOL/20.084

*Received for publication:

November 18, 2020

Accepted: December 17, 2020

Published: December 24, 2020

\begin{abstract}
Background: Angioleiomyoma is an uncommon, benign tumour originating from vascular smooth muscle. Occurrence in the nasal cavity, especially the nasal septum, is rare with only 16 cases of nasal septal angioleiomyoma reported thus far. We present a new case of nasal septal angioleiomyoma.
\end{abstract}

Case presentation: 52-year-old female presented with a three-week history of persistent epistaxis and nasal obstruction. Physical examination revealed a right sided nasal mass originating from the septum. The tumour was endoscopically excised and sent for pathological evaluation which revealed the diagnosis of angioleiomyoma. The patient was followed for six months postoperatively and showed no evidence of lesion recurrence.

Conclusions: Because nasal cavity angioleiomyoma recurrence has been reported due to incomplete tumour resection, wide marginal excision is necessary for successful treatment. There have been no cases of malignant transformation of nasal angioleiomyomas; however, malignant transformation at other sites has been reported. For these reasons, it is important to adequately biopsy and remove angioleiomyoma lesions.

Key words: angioleiomyoma, nasal septum, epistaxis

\section{Introduction}

Angioleiomyoma is an uncommon, benign smooth muscle growth that originates from the tunica media of vessels. Angioleiomyomas are seldom found in the nasal cavity with the first case being reported by Maesaka in $1966^{(1)}$. Since then, 68 additional cases have been reported worldwide. Of these cases, only 16 occurred on the nasal septum making this location exceedingly rare ${ }^{(2)}$. We present a new case of nasal septal angioleiomyoma, discuss the clinical and pathologic findings and review management. We additionally evaluate the role of sex hormones in the genesis of nasal angioleiomyoma.

This case study is aimed at bringing awareness to a rare vascular nasal neoplasm that causes common otolaryngology complaints such as epistaxis and nasal obstruction. It's consideration in the differential diagnosis is important for providing adequate patient treatment and preventing lesion recurrence.

\section{Case report}

A 52-year-old female presented to the clinic for evaluation of a three-week history of persistent epistaxis requiring anterior nasal packing in the emergency department. Her symptoms began after using allergy medications Fluticasone and Loratadine. Additional medical history included nasal obstruction and recurrent sinusitis.

Physical examination revealed a lesion in the right nasal cavity. Computed tomography (CT) showed a right mid nasal septal mass and nasal obstruction secondary to bilaterally enlarged inferior and middle turbinates (Figure 1and 2). Further cuts of the imaging demonstrated significant left septal deviation with narrowing and obstruction of the maxillary sinuses. Due to these issues, surgical intervention was recommended. 


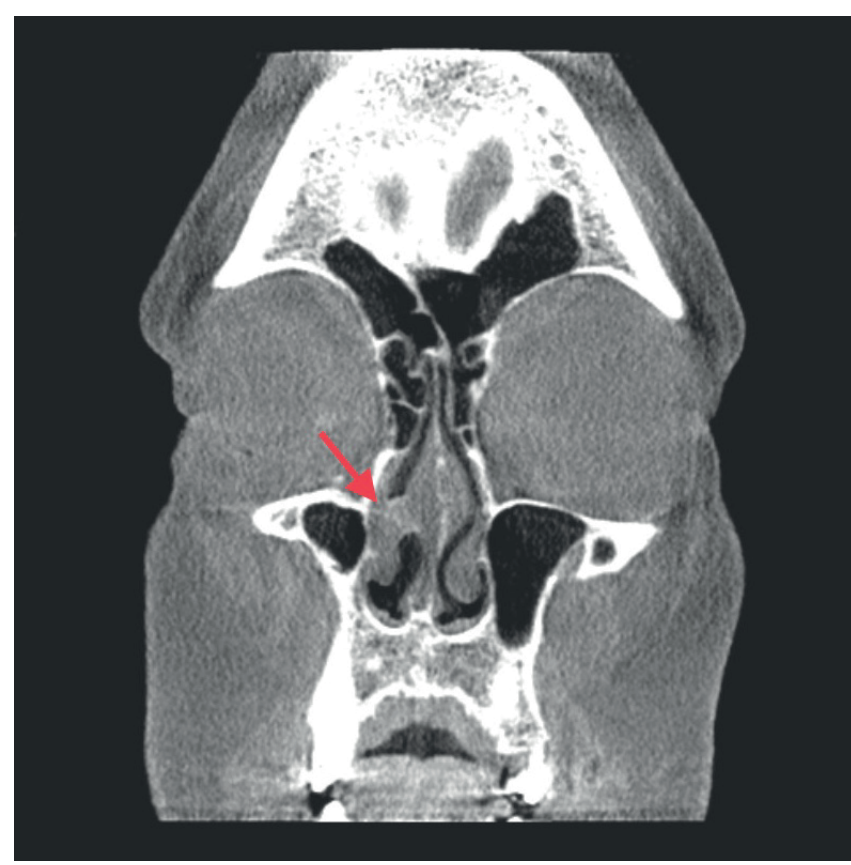

Figure 1. Non-contrast coronal computed tomography (CT) image showing a right nasal septal mass (depicted by the red arrow).

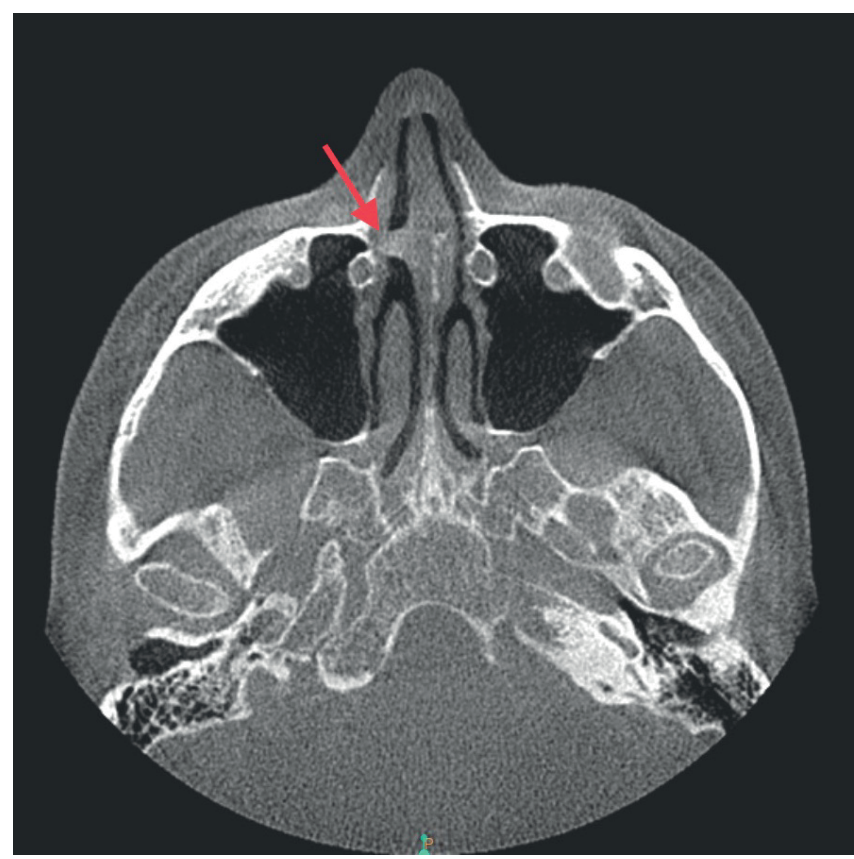

Figure 2. Non-contrast axial computed tomography (CT) image showing a right nasal septal mass (depicted by the red arrow).
The patient was scheduled for endoscopic sinus surgery and septoplasty with resection of the nasal mass under general anesthesia. Intraoperatively, the lesion was found to be an irregularly shaped, sessile mass originating from the right nasal septum and extending to the superior edge of the inferior turbinate. The base of the mass was pink and fleshy while the part contacting the inferior turbinate appeared glossy and erythematous (Figure 3). Excisional biopsy of the tumour with wide margins was performed before proceeding with the rest of the procedure. The specimen was sent for pathologic examination.

On pathology report, the gross specimen measured $0.4 \times 0.4 \mathrm{x}$ $0.2 \mathrm{~cm}$. Multiple sections were stained with hematoxylin and eosin (H\&E), and periodic acid-Schiff (PAS) staining was negative for fungi (Figure 4). Immunostaining for alpha smooth muscle actin $(\alpha-S M A)$ was positive and supported the diagnosis of angioleiomyoma (Figure 5).

The patient's postoperative course was complicated with only mild nasal bleeding. Monthly follow-ups for six months showed no evidence of nasal mass recurrence.

\section{Discussion}

Angioleiomyoma is a benign tumour originating from vascular smooth muscle and is typically found in the uterus (95\%), skin (3\%) and gastrointestinal tract (1\%). Nasal cavity angioleiomyomas make up only $0.5 \%$ of reported cases ${ }^{(3)}$. Manifestation in the nasal cavity is rare, most likely secondary to the scarcity of smooth muscle fibers in this location. Smooth muscle in the sinonasal tract can be found in the form of blood vessel tunica media, piloerector muscle located in the anterior vestibule and turbinate contractile tissue ${ }^{(4)}$. In the nose, inferior turbinates are most commonly affected followed by the nasal septum and vestibule.

The literature shows a female predilection with a female to male ratio of 2.7:1 ${ }^{(5)}$. Middle aged women are most commonly affected, and the mean age is $54.5^{(2)}$. One possible explanation for the female predominance is the presence of progesterone and estrogen receptors on nasal angioleiomyomas. Zhu et al. reported a study where five out of six patients with nasal angioleiomyomas expressed progesterone and estrogen receptors in the nuclei of the smooth muscle tumour cells on pathological examination ${ }^{(6)}$. Marioni et al. also presented a case of nasal cavity angioleiomyoma which was found to be progesterone receptor positive ${ }^{(7)}$. Our patient was not tested for these receptors. While it is suggested that the growth of this tumour may be hormone dependent, more large scale studies are needed to explore the effects of progesterone and estrogen.

Nasal angioleiomyoma is a slow growing tumour that commonly presents with nonspecific symptoms such as epistaxis and nasal obstruction ${ }^{(1-7)}$. Less common presentations include headache, facial pain and nasal discharge. Our patient presented with epistaxis and nasal obstruction, and she also reported a history of recurrent sinusitis. Chronic sinusitis has not been described in any other case. Computed tomography (CT) imaging is nonspecific and not sufficient to diagnose angioleiomyoma; 


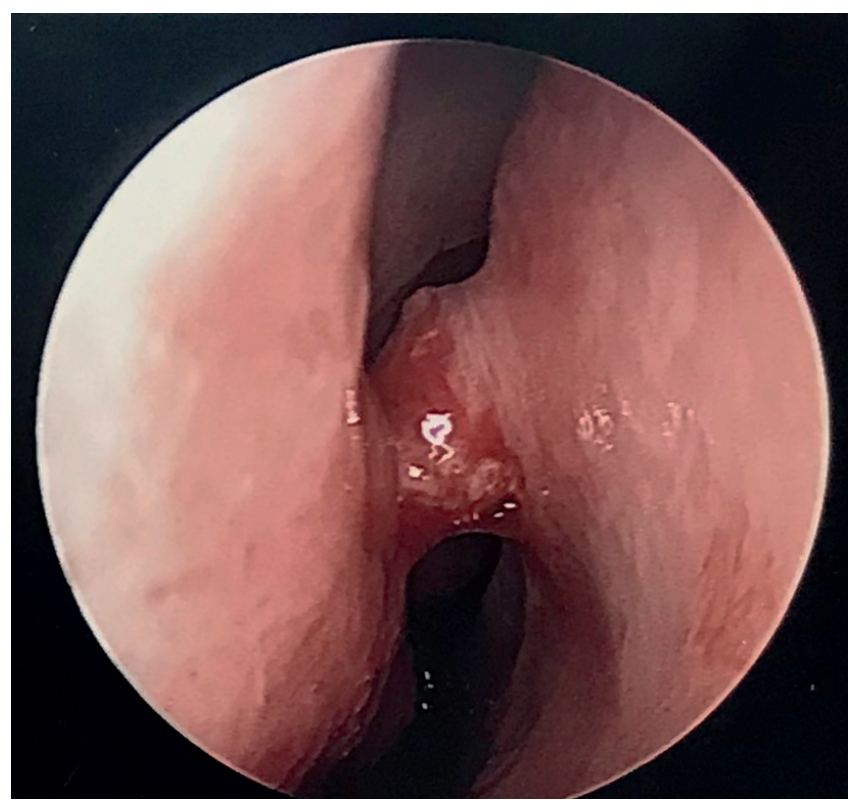

Figure 3. Endoscopic photograph of the right nasal cavity showing a 0.4 $x 0.4 \times 0.2 \mathrm{~cm}$ angioleiomyoma originating from the septum and extending to the superior edge of the inferior turbinate.

however, it can be helpful in visualizing the extent of tumour growth to better plan appropriate treatment ${ }^{(5)}$.

Due to nonspecific clinical and imaging evaluation, the provider needs to form a broad differential diagnosis including haemangioma, hemangiopericytoma, angiofibroma, leiomyoblastoma, angiosarcoma and leiomyosarcoma ${ }^{(3,8)}$. Histopathological examination is necessary to establish the definitive diagnosis of angioleiomyoma. Characteristic histopathological features include well differentiated smooth muscle cell bundles surrounding blood vessel lumina with elongated cigar shaped nuclei. Cellular pleomorphism and mitotic figures are absent. Additionally, angioleiomyomas strongly express alpha smooth muscle actin, h-caldesmon and muscle-specific actin by immunohistochemistry staining ${ }^{(9)}$. Histologic examination of our patient's septal tumour demonstrates proliferation of smooth muscle cells surrounding blood vessels with positive straining for alpha smooth muscle actin (Figure 4 and 5). No cellular atypia, cellular pleomorphism or mitotic figures were seen.

\section{Conclusions}

Treatment of choice is complete removal of the nasal mass, and the surgical approach will depend on the size and extent of the lesion. Small angioleiomyomas confined to the nasal cavity can be managed with endoscopic excision, as was done for our patient. Large tumours extending to the nasopharynx or cranial vault may require craniofacial resection ${ }^{(10)}$. It is critical to achieve wide marginal excision of the tumour, because lesion recurrence

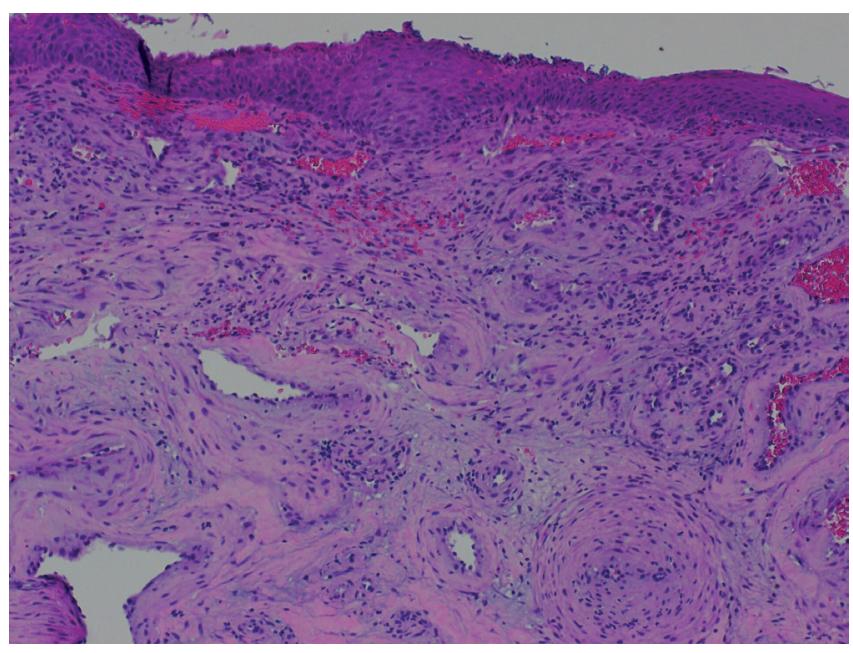

Figure 4. Hematoxylin and eosin staining (x200) showing smooth muscle cell proliferation with thickened blood vessel walls.

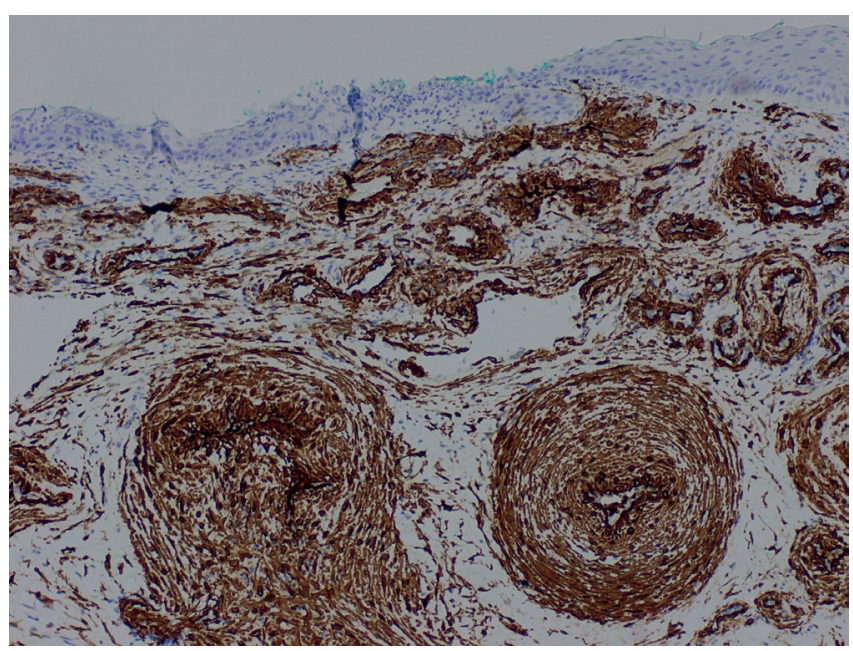

Figure 5. Histopathologic image showing positive immunostaining for alpha smooth muscle actin.

has been reported secondary to incomplete primary excision in three separate cases ${ }^{(3,11,10)}$. While there is no evidence of malignant transformation of angioleiomyoma in the nasal cavity, it has been reported in other locations such as the hands ${ }^{(12,13)}$. For these reasons, it is important to appropriately biopsy and excise nasal lesions suspicious for angioleiomyoma.

\section{Acknowledgments}

Not applicable

\section{Authorship contribution}

GN wrote the manuscript and contributed to review of the literature. SK contributed to the review of the literature, collected patient information and supervised. AH provided histopathologic images. All authors read and reviewed the final manuscript. 


\section{Conflict of interest}

The authors declare that they have no competing interests.

\section{Funding}

Not applicable

\section{Consent for publication}

Written informed consent for publication of clinical details and clinical images was obtained from the patient.

\section{Availability of data and materials}

Not applicable.

\section{References}

1. Maesaka A, Keyaki Y, Nakahashi T. Nasal angioleiomyoma and leiomyosarcomareport of 2 cases. Otologia Fukuoka. 1966; 12: $42-7$.

2. Varadarajan VV, Justice JM Angioleiomyoma of nasal septum: Case report and literature review. Otolaryngol Case Rep, 2016; 1: 1-4.

3. Bhandarkar AM, Ramaswamy B, Jaiprakash P, Chidambaranathan N. Recurrent angioleiomyoma of the middle turbinate. BM Case Rep. 2015 Aug 3;2015:bcr2015210686.

4. Agarwal AK, Bansal R, Singhal D. Sinonasa Leiomyoma: Report of 2 Cases. Ear Nose Throat J. 2005; 84: 224-230.

5. Mathieu T, Verbruggen A, Goovaerts G, Declau F. Vascular Leiomyoma of the Nasal Cavity: Case Report and Literature Review. Eur Arch Otorhinolaryngol. 2016; 274: 579583.

6. Zhu G, Xiao D, Sun P. Expression of Estrogen and Progesterone Receptors in Angioleiomyoma of the Nasal Cavity of Six
Patients. Oncol Lett. 2016; 11: 2359-2364.

7. Marioni G, Marchese-Ragona $R$ Fernandez S, Bruzon J, Marino F, Staffier A. Progesterone Receptor Expression in Angioleiomyoma of the Nasal Cavity. Acta Otolaryngol. 2002; 122: 408-412.

8. Fu Y, Perzin K. Non-Epithelial Tumors of the Nasal Cavity, Paranasal Sinuses, and Nasopharynx: A Clinicopathologic Study. Cancer 1974; 33: 1275-1288.

9. Agaimy A, Michal M, Thompson LDR, Michal M. Angioleiomyoma of the Sinonasal Tract: Analysis of 16 Cases and Review of the Literature. Head Neck Pathol. 2015; 9: 463473.

10. Nicolai P, Redaeilli de Zinis L, Facchett F, Maroldi R, Antonelli A. Craniofacia Resection for Vascular Leiomyoma of the Nasal Cavity. Am J Otolaryngol 1996; 17 340-344.

11. Bloom D, Finley JC, Broberg TG, Cueva RA. Leiomyoma of the Nasal Septum. Rhinology 2001; 39: 233-235.

12. Kim AY, Choi MS, Jang DS, Lee HY, A Rare
Case of Intranasal Vascular Leiomyoma. BM Case Rep. 2015 Jun 10;2015:bcr2014208247.

13. Herren D, Zimmermann A, Buchler U Vascular Leiomyoma in an Index Finger Undergoing Malignant Transformation. J Hand Surg Br.1995; 20: 484-487.

\section{Gabriele Noreikaite}

Midwestern University

Chicago College of Osteopathic

Medicine

Downers Grove

IL, USA

Tel: +1 (630) 999-1007

E-mail:

gnoreikaite36@midwestern.edu 